\title{
Characterization of Prognostic Factors and the Efficacy of Adjuvant S-1 Chemotherapy in Patients with Post-surgery Extrahepatic Bile Duct Cancer
}

\author{
TAKEHIRO OKABAYASHI ${ }^{1}$, YASUO SHIMA ${ }^{1}$, JUN IWATA $^{2}$, SOJIRO MORITA $^{3}$, \\ TATSUAKI SUMIYOSHI ${ }^{1}$, KENTA SUI $^{1}$, YASUHIRO SHIMADA ${ }^{4}$ and TATSUO IIYAMA ${ }^{5}$ \\ ${ }^{1}$ Department of Gastroenterological Surgery at Kochi Health Sciences Center, Kochi, Japan; \\ ${ }^{2}$ Department of Diagnostic Pathology at Kochi Health Sciences Center, Kochi, Japan; \\ ${ }^{3}$ Department of Radiology at Kochi Health Sciences Center, ${ }^{4}$ Department of Clinical Oncology, and \\ ${ }^{5}$ Department of Biostatistics at Kochi Medical School, Kochi, Japan
}

\begin{abstract}
Background/Aim: There is no clear consensus on the type of adjuvant therapy that should be used for patients with extrahepatic bile duct cancer. Patients and Methods: Two hundred and seventy-one patients that had undergone surgical resection for extrahepatic bile duct cancer composed the study cohort. Demographics, treatments, and relationships between the potential prognostic factors and survival rates were analyzed. Results: The overall 3-year and 5-year survival rates for post-surgery extrahepatic bile duct cancer patients were $49.0 \%$ and $35.4 \%$, respectively. Multivariate analysis revealed that regional lymph node metastasis was an independent negative prognostic factor. We observed a significant correlation between node-positive extrahepatic bile duct cancer and postoperative local recurrence, liver metastasis, peritoneal dissemination, and post-surgery lymph node metastasis. Adjuvant S-1 chemotherapy showed a favorable hazard ratio in patients with lymph node metastases or positive vascular invasion. Conclusion: We recommend the use of adjuvant S-1 therapy in patients with lymph node metastases or microvascular invasion.
\end{abstract}

Cholangiocarcinomas are a very heterogeneous and rare group of neoplasms that originate from the intrahepatic, perihilar, or extrahepatic bile duct epithelium. Although they represent only $3 \%$ of gastrointestinal cancers, their incidence

Correspondence to: Takehiro Okabayashi, MD, Ph.D., Department of Gastroenterological Surgery, Kochi Health Sciences Center, 2125-1 Ike, Kochi-City, Kochi 781-8555, Japan. Tel: +81 888373000, Fax: +81 888376766, e-mail: tokabaya@gmail.com

Key Words: Extrahepatic bile duct cancer, prognosis, surgery, adjuvant chemotherapy, $\mathrm{S}-1$. is increasing (1). According to the staging system (7th edition) developed by the Union for International Cancer Control (UICC) and the American Joint Committee on Cancer (AJCC), extrahepatic bile duct cancers (EBDCs) comprise perihilar-type and distal-type tumors (2).

Surgical resection remains the only curative treatment option for EBDCs. Unfortunately, the rate of resectability for these tumors is quite low. Furthermore, patient prognosis after curative resection is poor, with a reported 5-year survival rate that ranges between $12 \%$ and $54 \%$, despite the use of aggressive surgical procedures such as major hepatectomies, pancreaticoduodenectomies, and extensive lymphadenectomies $(3,4)$. This poor prognosis is attributed to high incidence of local recurrence and the development of distant metastases. Due to the high rate of disease recurrence and poor survival rates following surgical resection, postoperative treatment modalities have been suggested to improve patient survival after EBDC resection (5). Randomized data on the matter is scarce, and only 2 randomized clinical trials have been conducted that have examined the efficacy of adjuvant chemotherapy after EBDC resection $(6,7)$. However, the results of these trials did not show an increase in survival for patients with EBDC, who had received chemotherapy. Despite this, evidence still supports the use of chemotherapy as an adjuvant approach for treating node-positive disease. Clarifications remain on the kind of chemotherapy, provided it has a proven impact on local disease control, improve overall survival, and whether the use of an agent in the adjuvant setting can influence patient outcome.

In the current study, we retrospectively clarified the clinicopathological features, outcomes, and prognostic factors of postoperative EBDC patients. Additionally, we evaluated whether postoperative adjuvant chemotherapy using S-1 is clinically viable as a treatment option for resectable EBDCs. 


\section{Patients and Methods}

Patients. EBDC diagnosis was initially performed after imaging and was further confirmed by pathological analysis. We retrospectively reviewed the surgical pathology database of the Kochi Health Sciences Center to identify patients who had undergone EBDC resections between April 1986 and December 2015. Clinical characteristics evaluated included age, gender, the part of the tumor, operative procedures, pathological data, and postoperative chemotherapy. Location of the EBDC, the degree of differentiation, vascular invasion, lymphatic permeation, perineural invasion, adjacent organ invasion, portal vein invasion, hepatic artery invasion, and lymph node metastasis were assessed according to the UICC/AJCC TNM staging system (2). Our department followed the prognosis for each case and obtained accurate details of the patients' outcome. Our cohort included patients with ordinary invasive ductal carcinomas of both the proximal and distal extrahepatic bile ducts, and excluded patients with adenocarcinomas originating from the intrahepatic bile duct, the cystic duct, and the gallbladder. This study was approved by the ethics committee of the Kochi Health Sciences Center. All patients provided written informed consent.

Adjuvant S-1 treatment for EBDC. Postoperative adjuvant chemotherapy using S-1 was introduced to our department in 2007, in order to assess its utility. Postoperative patients allocated to S-1 adjuvant chemotherapy received oral S-1 twice a day, from Day 1 to 14 of the 21-day treatment cycle, and at a dose according to their body-surface area (BSA) $\left(<1.25 \mathrm{~m}^{2}, 60 \mathrm{mg} /\right.$ day; $\geq 1.25$ to $<1.5 \mathrm{~m}^{2}$, $80 \mathrm{mg} /$ day; $\geq 1.5 \mathrm{~m}^{2}, 100 \mathrm{mg} /$ day). All patients received adjuvant $\mathrm{S}$ 1 chemotherapy within 2 months of their curative surgical resection, and this was continued for as long as possible.

Assessments. The study data was prospectively collected and retrospectively analyzed by a biostatistician (T.I.). Overall survival defined as the time between tha date of surgical resection and date of death from any cause. The primary endpoint of this study was to investigate overall survival in EBDC patients after they had been treated by surgery with the intent-to-cure and to evaluate postoperative prognostic factors. The secondary endpoint was to evaluate whether postoperative adjuvant chemotherapy using S-1 was clinically viable for treating resectable EBDCs.

Statistics. Survival curves were generated via the Kaplan-Meier method and compared using the log-rank test (8). Patients alive as of December 31, 2015 were censored at the time of follow-up. Continuous variables were presented as a median \pm standard deviation. Data were analyzed using the Student's $t$-test (two-tailed), with dichotomous variables analyzed by either the chi-square test (twotailed) or the Fisher's exact test (two-tailed). A multivariate Cox regression analysis identified the factors that were independently associated with EBDC-related death (9). The correlation between the identified prognostic factors and the incidence of postoperative recurrence was evaluated by calculating the Spearman correlation coefficient. A $p$-value $<0.05$ was considered significant. All analyses were performed using SPSS ${ }^{\circledR}$ (SPSS; Chicago, IL, USA).

\section{Results}

Patients. We studied 271 patients with EBDC who were operated between April 1986 and December 2015 at the
Table I. Characteristics of patients who underwent surgical resection for carcinoma of the extrahepatic bile duct.

\begin{tabular}{lccc}
\hline Characteristic & $\begin{array}{c}1986-2002 \\
(\mathrm{n}=114)\end{array}$ & $\begin{array}{c}2003-2015 \\
(\mathrm{n}=157)\end{array}$ & $p$-Value \\
\hline Gender (male/female) & $79 / 35$ & $103 / 54$ & 0.523 \\
Age & $70 \pm 8$ & $74 \pm 9$ & $<0.001$ \\
Total bilirubin & $8.5 \pm 7.4$ & $4.0 \pm 6.2$ & $<0.001$ \\
CEA & $1.7 \pm 3.0$ & $2.7 \pm 3.6$ & 0.020 \\
CA19-9 & $80.8 \pm 22871.0$ & $82.4 \pm 574.5$ & 0.204 \\
Main site of the tumor (Bp/Bd)* & $63 / 51$ & $66 / 91$ & 0.031 \\
Type of operation & & & \\
LR/BDR/PD/HPD & $41 / 12 / 47 / 14$ & $47 / 26 / 82 / 2$ & 0.002 \\
Mortality (\%) & $15(13.2)$ & $5(3.2)$ & 0.004 \\
Pathological findings & & & \\
Well/Mod/Por & $50 / 58 / 6$ & $66 / 76 / 15$ & 0.571 \\
Superficial spreading lesions (\%) & $43(37.7)$ & $74(47.1)$ & 0.800 \\
Portal vein invasion (\%) & $26(22.8)$ & $19(12.1)$ & 0.005 \\
Hepatic arterial invasion (\%) & $17(14.9)$ & $12(7.6)$ & 0.087 \\
Adjacent organs (\%) & $68(59.6)$ & $88(56.1)$ & 0.640 \\
Perineural invasion (\%) & $31(46.2)$ & $83(52.9)$ & 0.634 \\
Microvascular invasion (\%) & $41(45.1)$ & $73(46.4)$ & 0.757 \\
Lymphatic permeation (\%) & $58(66.7)$ & $73(46.8)$ & 0.003 \\
Lymph node metastases (\%) & $48(42.1)$ & $54(34.8)$ & 0.225 \\
Resection margin (\%) & $53(46.5)$ & $47(30.3)$ & 0.006 \\
Adjuvant therapy with S-1 (\%) & $0(0.0)$ & $47(30.3)$ & $<0.001$ \\
\hline
\end{tabular}

*Main site of the tumor was classified according to to the 7th edition of staging system developed by the Union for International Cancer Control and the American Joint Committee on Cancer. CEA: Carcinoemboryonic antigen, CA19-9: carbohydrate antigen 19-9, Bp: proximal bile duct, Bd: distal extrahepatic bile ducts, LR: major liver resection with biliary reconstruction, BDR: bile duct resection, PD: pancreaticoduedenectomy, HPD: hepatopancreatoduodenectomy, Well: well-differentiated adenocarcinoma, Mod: moderately differentiated adenocarcinoma, Por: pooly differentiated adenocarcinoma.

Kochi Health Sciences Center. Our patient cohort was composed of 182 men and 89 women, with patients age ranging from 42 to 92 years (mean 70.8) (Table I). Since 2003, EBDC treatment has been carried out by a fixed team of medical experts, including gastroenterologists, surgeons, radiologists, oncologists, and pathologists. We evaluated postoperative clinicopathological features of EBDCs and patients were divided into 2 groups: the "past group" composed of patients who were treated between 1986 and 2002, and the "recent group" composed of patients treated between 2003 and 2015. Median age in the recent group (74 \pm 9 years; range $=42-90$ years $)$ was significantly higher than in the past group $(70 \pm 8$ years; range $=47-92$ years $)$. Furthermore, there were significant differences between the 2 groups in terms of tumor location, surgery type, and pathological findings (Table I). The incidence of pathological portal vein invasion $(22.8 \%$ in the past group $v s .12 .1 \%$ in the recent group) and lymphatic permeation $(66.7 \%$ in the 


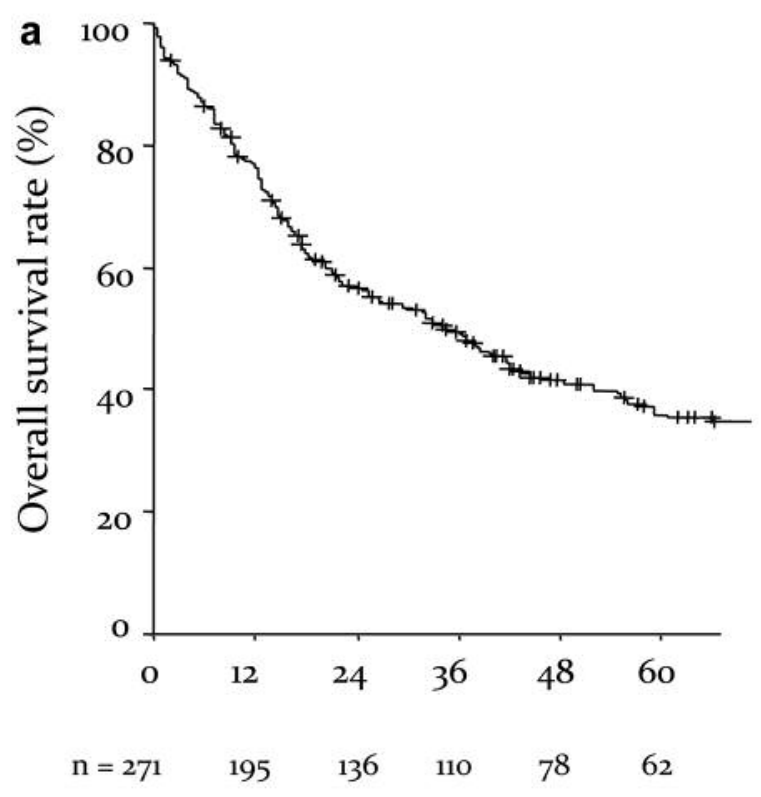

Months after surgery

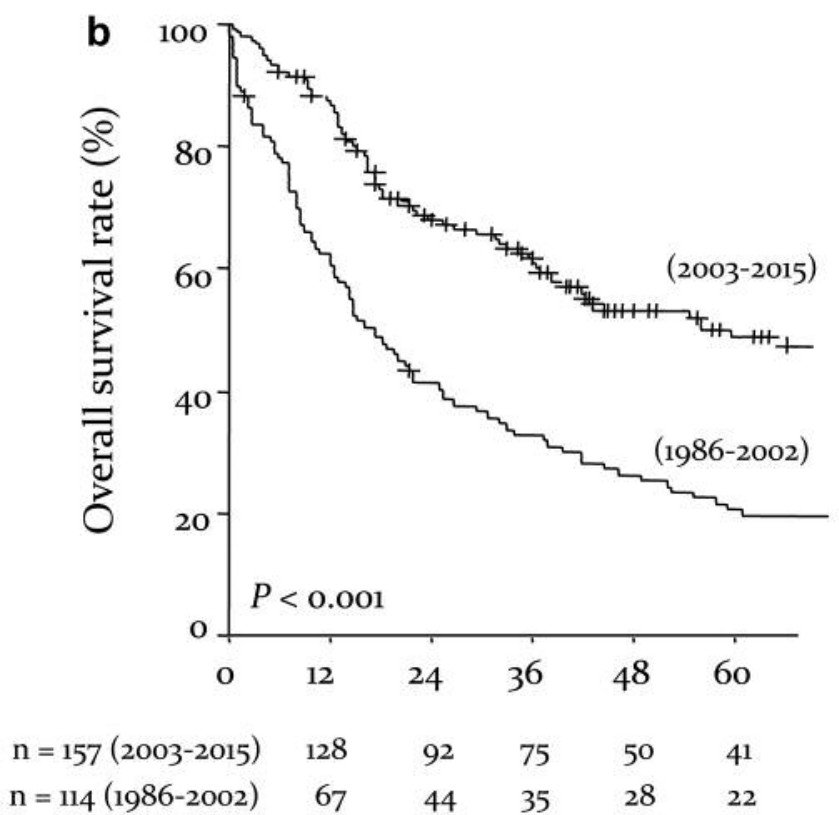

Months after surgery

Figure 1. Kaplan-Meier estimates of overall survival (A) and survival compared to changing trends $(B)$.

past group $v s .46 .8 \%$ in the recent group) was significantly in the past group. These data suggest that the more aggressive surgical approaches, used to treat advanced EBDCs, were previously performed by unexperienced staff, resulting in the higher mortality rate $(13.2 \%$ in the past group vs. $3.2 \%$ in the recent group) and the increased rate of positive resection margins $(46.5 \%$ in the past group $v s$. $30.3 \%$ in the recent group), when compared to the recent group. The frequency of adjuvant S-1 was significantly higher in the recent group, as postoperative adjuvant chemotherapy using S-1 was introduced to our department only after 2007 (Table I). Patients received adjuvant S-1 chemotherapy immediately after curative surgical resection, and chemotherapy using S-1 oral administration was continued as long as possible. Median treatment duration, in the 47 patients that received adjuvant S-1 treatment, was 15.7 months (range=2.4-45.7 months).

Survival. Patient follow-up, as of December 2015, ranged from 1.0 to 144.5 months, with a median of 24.4 months (mean, 37.8 months). Of the 271 patients, 169 (62.4\%) died, forming the basis for our analysis of overall survival. Overall 1-year, 3-year, and 5-year survival rates after surgery were $76.2 \%, 49.0 \%$, and $35.4 \%$, respectively (Figure 1a). Median overall survival for patients who underwent curative surgical resection for EBDC was 34.2 months. As expected, overall long-term survival rates were significantly in the past group (Figure 1b). Overall 1-year, 3-year, and 5-year postoperative survival rates in the past group were $61.5 \%, 32.9 \%$, and $20.7 \%$, respectively. In contrast, 1-year, 3-year, and 5-year post-surgery survival rates for the recent group were $86.8 \%$, $61.1 \%$, and $48.7 \%$, respectively (Figure 1b). Median overall survival was 55.8 months in the recent group and 17.3 months in the past group $(p<0.001)$. When comparing survival rates between the subgroups identified by each predictive factor, the following factors were found to be significantly associated with poor post-surgery outcomes: 1) the presence of tumor invasion into the adjacent organs, 2) the presence of superficial spreading lesion from the main EBDC, 3) the presence of portal vein invasion, 4) the presence of hepatic artery invasion, 5) a positive surgical margin, 6) the presence of perineural invasion, 7) the presence of lymphatic permeation, 8) the presence of microvascular involvement, and 9) positive lymph node metastasis (Table II). Our pathological assessments of EBDC patients after surgical resection with the intent-to-cure suggested that patients displaying hepatic artery invasion have a high risk of being classified falsely as having unresectable disease. In multivariate analysis, the presence of metastatic lymph nodes was found to be independently associated with poor survival (Table II). Although postoperative pathological values for the presence of tumor 
Table II. Univariate and multivariate analysis revealed the following factors to be independently associated with poor survival.

\begin{tabular}{|c|c|c|c|c|c|c|}
\hline \multirow[t]{2}{*}{ Variable } & \multirow[t]{2}{*}{$\mathrm{N}$} & \multicolumn{2}{|c|}{ Overall survival } & \multirow{2}{*}{$\begin{array}{c}\text { Univariate, } \\
p \text {-Value }\end{array}$} & \multirow{2}{*}{$\begin{array}{c}\text { Multivariate } \\
\text { Risk ratio }(95 \% \mathrm{CI})\end{array}$} & \multirow[t]{2}{*}{$p$-Value } \\
\hline & & 3 -yrs $(\%)$ & 5 -yrs $(\%)$ & & & \\
\hline Tumor location & & & & 0.052 & & \\
\hline $\mathrm{Bp}$ & 142 & 43.5 & 28.2 & & & \\
\hline $\mathrm{Bd}$ & 129 & 54.4 & 43.0 & & & \\
\hline Differentiation & & & & 0.417 & & \\
\hline Well differentiated & 134 & 52.3 & 39.8 & & & \\
\hline Moderately differentiated & 116 & 41.2 & 28.1 & & & \\
\hline Poorly differentiated & 21 & 47.2 & 38.6 & & & \\
\hline Tumor invasion into the adjacent organs & & & & 0.005 & & 0.743 \\
\hline Absent & 111 & 59.8 & 47.6 & & 1 & \\
\hline Present & 142 & 41.0 & 27.7 & & $1.123(0.560-2.253)$ & \\
\hline Superficial spreading infiltration & & & & 0.008 & & 0.321 \\
\hline Absent & 154 & 54.8 & 40.2 & & 1 & \\
\hline Present & 117 & 41.7 & 30.8 & & $1.419(0.711-2.832)$ & \\
\hline Portal vain invasion & & & & 0.001 & & 0.075 \\
\hline Negative & 226 & 54.1 & 40.2 & & 1 & \\
\hline Positive & 43 & 23.1 & 13.2 & & $3.159(0.889-11.230)$ & \\
\hline Hepatic artery involvement & & & & $<0.001$ & & NA \\
\hline Negative & 241 & 53.2 & 40.1 & & NA & \\
\hline Positive & 28 & 14.1 & 0.0 & & NA & \\
\hline Surgical resection margin & & & & 0.002 & & 0.403 \\
\hline Negative & 162 & 53.9 & 41.3 & & 1 & \\
\hline Positive & 91 & 40.9 & 27.0 & & $1.368(0.656-2.854)$ & \\
\hline Peri-neural invasion & & & & 0.007 & & 0.227 \\
\hline Negative & 120 & 69.5 & 60.2 & & 1 & \\
\hline Positive & 114 & 48.6 & 35.3 & & $1.557(0.759-3.195)$ & \\
\hline Lymphatic permeation & & & & 0.001 & & 0.676 \\
\hline Negative & 111 & 62.9 & 51.1 & & 1 & \\
\hline Positive & 131 & 40.3 & 28.2 & & $1.187(0.532-2.647)$ & \\
\hline Microvascular invasion & & & & 0.001 & & 0.262 \\
\hline Negative & 132 & 70.6 & 58.7 & & 1 & \\
\hline Positive & 114 & 39.1 & 28.0 & & $1.550(0.721-3.334)$ & \\
\hline Lymph node metastasis & & & & 0.001 & & 0.026 \\
\hline Negative & 155 & 62.8 & 51.1 & & 1 & \\
\hline Positive & 98 & 27.0 & 12.3 & & $2.584(1.075-6.211)$ & \\
\hline Adjuvant treatment & & & & 0.042 & & 0.284 \\
\hline S-1 usage & 47 & 58.4 & 49.5 & & 1 & \\
\hline Observation & 206 & 46.9 & 33.8 & & $1.684(0.743-3.817)$ & \\
\hline
\end{tabular}

Bp: Proximal bile duct, Bd: distal extrahepatic bile ducts, 95\% CI: 95\% confidence interval, NA: not available.

invasion into the adjacent organs, the presence of a superficial spreading lesion from the main EBDC, the presence of portal vein invasion, a positive surgical margin, the presence of perineural invasion, the presence of lymphatic permeation, and the presence of microvascular involvement were significant prognostic factors in a univariate analysis, these factors were not found to be significant in multivariate analysis. Notably, our data suggested that postoperative adjuvant $\mathrm{S}-1$ treatment might be clinically beneficial in postsurgery EBDC patients, as median overall survival in the observation group was 31.6 months and 41.9 months in the S-1 setting group $(p=0.042)$ (Table II).
Recurrence. Recurrence was not evaluated in the 18 patients who suffered in-hospital mortalities; 10 patients died due to unrelated diseases; 3 , due to other organ cancers (1 intrahepatic cholangiocarcinoma, 1 pancreatic carcinoma, and 1 carcinoma of the urinary tract); and 2, lost to follow-up. Of the remaining 271 patients, $100(36.9 \%)$ patients showed no recurrence. The major sites of recurrence were the resection stump (70 patients), the liver (38 patients), the peritoneal dissemination (37 patients), and the lymph node (16 patients). The median time to the event for recurrence was 12.3 months (range, 1.4 to 77.3 months). The time to recurrence seemed to be shorter when tumor recurrence was spotted in the liver (Table III). We 
Table III. Pattern of recurrence after surgery.

\begin{tabular}{lcc}
\hline Location of recurrence & $\begin{array}{c}\text { No. of } \\
\text { patients }\end{array}$ & $\begin{array}{c}\text { Time to recurrence } \\
\text { Median (range) (mo) }\end{array}$ \\
\hline Over all & $136^{*}$ & $12.3(1.4-77.3)$ \\
Local recurrence & 70 & $17.5(3.1-53.9)$ \\
Liver metastases & 38 & $7.7(2.4-29.0)$ \\
Peritoneal dissemination & 37 & $12.2(1.5-77.3)$ \\
Lymph node metastases & 16 & $10.0(3.5-23.0)$ \\
Lung metastases & 9 & $13.1(1.4-93.8)$ \\
Bone metastases & 1 & 3.0 \\
\hline
\end{tabular}

*Eighteen patients of in-hospital death, 10 of death of other diseases, 3 of death of other organ cancer (1 intrahepatic cholangiocarcinoma, 1 pancreatic carcinoma, and 1 in carcinoma of the urinary tract), and two who were lost to follow up were excluded for analysis were excluded for analysis. One hundred patients had no recurrence.

found a significant association between patients with pathologically node-positive EBDCs and patients group displaying incidence of local recurrence, liver metastases, peritoneal dissemination, and lymph node metastases (Table III). As expected, there was a significant correlation between microvascular invasion and liver metastasis and an association between lymphatic permeation and lymph node metastasis. Interestingly, when patients were displaying a superficial spreading infiltration, a positive resection margin, or a postsurgical portal vein invasion, the occurrence of postoperative peritoneal dissemination was significantly higher than in patients displaying none of these conditions (Table IV).

Subgroup analysis. Subgroup analyses of survival, according to postoperative pathological characteristics showed significant differences between the observation group and the adjuvant S-1 group (Table II). On forest plots of S-1 treatment effects on overall survival, the adjuvant $S-1$ group showed a favorable HR, when compared with the observation group, in the subset of patients who displayed positive vascular invasion and lymph node metastasis. However, S-1 failed to improve overall patient survival at a statistically significant level (HR, 0.59; 95\% CI, 0.26 to $1.34 ; p=0.284$ ) (Figure 2).

\section{Discussion}

We identified the clinicopathological characteristics of EBDC and determined the relationships between potential prognostic factors and postoperative survival. The only potentially curative treatment option for patients who have a resectable disease is surgery. However, for other patients, 5year survival rate is only around $12-54 \%(3,4,10)$. In our study, the overall 5-year survival rate for earlier patients was
$20.7 \%$, with an in-hospital mortality rate of $13.2 \%$. These were significantly worse than the corresponding figures in more contemporary patients, where the 5-year survival rate was $48.7 \%$, and the in-hospital mortality rate $3.2 \%$. We should note, that overall survival in our recent group was comparable to the figure seen other reports $(3,4,10,11)$. This might be due to a shift in patient recruitment, as in 2003, perihilar carcinomas, which involve the intra hepatic bile duct, were excluded by our fixed expert medical team. Based on previous experience, we know that the complete resection of tumors with pathologically-negative margins is difficult, due to their deep location and adjacency to critical organs, especially when EBDCs are at an advanced stage. In our department, resections involving intrahepatic- and extrahepatic bile ducts and those associated with the hepatic and caudate lobe, are the standard of care for suitable patients, although a fair number of patients have unresectable EBDCs $(12,13)$. In contrast, any of the following criteria categorize a non-metastatic hilar cancer as unresectable: (1) bilateral segmental ductal extension; (2) unilateral atrophy with either contralateral segmental ductal or vascular inflow involvement, (3) unilateral segmental ductal extension with contralateral vascular inflow involvement, and (4) the requirement for a hepatic arterial reconstruction induced by EBDC invasion. Obviously, because of improvements in preoperative diagnostic technology, surgical techniques, and perioperative care, patients treatment has dramatically ameliorated in recent years, thereby reducing the mortality rate and improving postoperative outcomes. However, the incidence of EBDCs is increasing and will continue to increase as the average population age rises $(3,14,15)$.

Although several prognostic factors have been reported for EBDCs, such as surgical radicality, nodal status, tumor invasion into the adjacent organs, differentiation, perineural invasion, and lymphovascular invasion (4, 16-20), the prognostic values of these factors are inconsistent. Among the aforementioned factors, only nodal metastasis, adjacent organ involvement, and positive surgical margin have been widely recognized to be associated with long-term survival (16-20). In regards to nodal metastases, the incidence of lymph node metastasis has been reported to range from 24-68\%, and lymph node involvement has been identified to be one of the most important predictors of survival in EBDC patients (19, 21-24). In our data, the frequency of lymph node metastasis was $37.6 \%$ and patients with nodal metastases showed worse survival rate in univariate analysis. Furthermore, the presence of a metastatic lymph node was found to be independently associated with poor survival in multivariate analysis. In this study, we achieved a relatively high rate of survival in patients with node-negative EBDCs, with a 5-year survival rate of $51.1 \%$. Nonetheless, long-term prognosis still remains unsatisfactory for postsurgical EBDC patients with lymph node metastases (5-year survival rate, 12.3\%). This is due to 


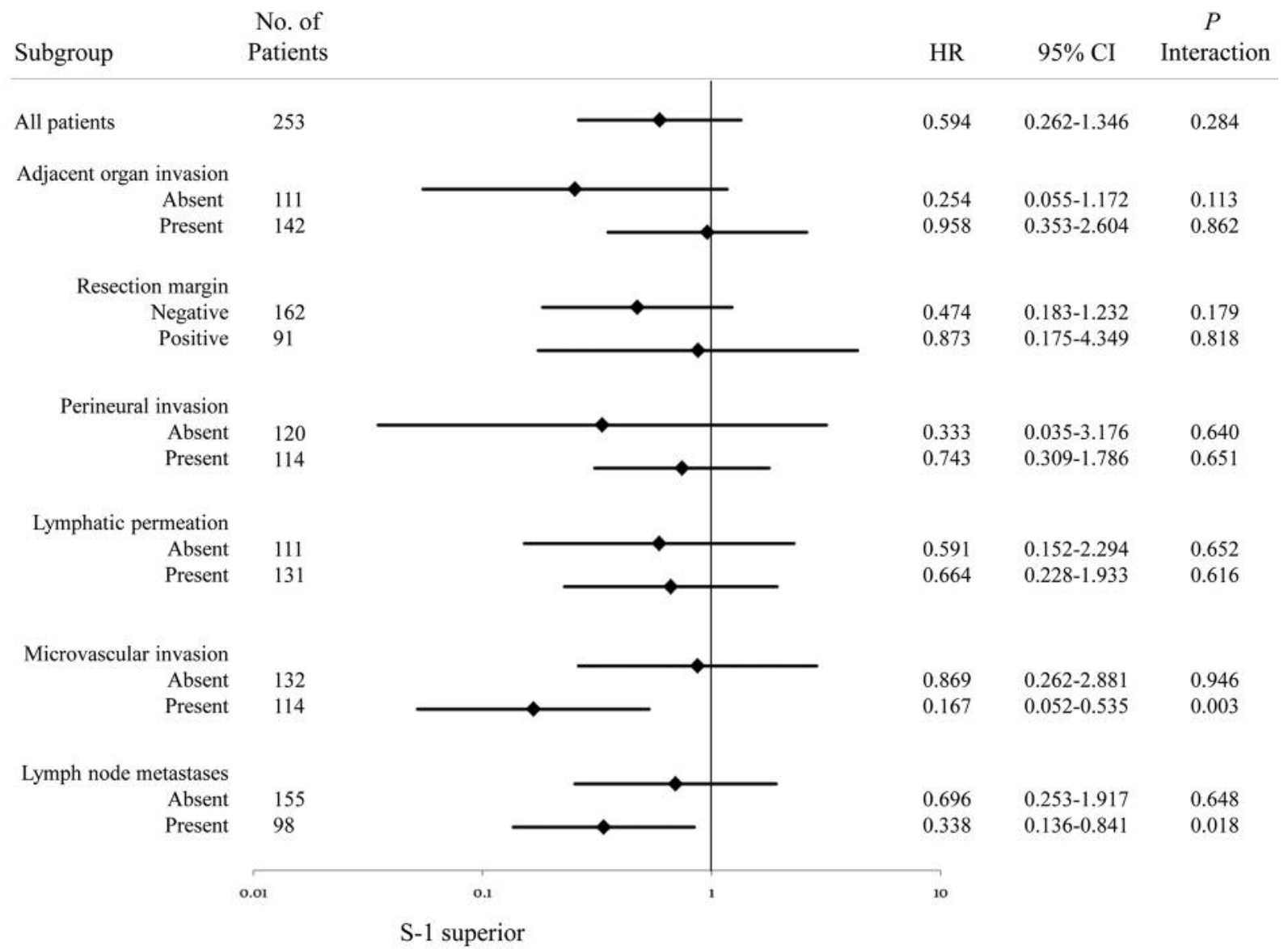

Figure 2. Forest plots of treatment effects on overall survival in subgroup analyses. Forest plots show effects on overall survival of patients in each group. Each black rhombus shows the treatment response.

Table IV. Correlation between poor prognostic factors and patterns of recurrence after surgery.

\begin{tabular}{|c|c|c|c|c|c|}
\hline \multirow[b]{2}{*}{ Characteristics } & \multicolumn{5}{|c|}{ Recurrence pattern } \\
\hline & Local & Liver & Peritoneal & Lymph nodes & Lung \\
\hline \multirow[t]{2}{*}{ Adjacent organ invasion } & $\mathrm{r}=0.016$ & $\mathrm{r}=0.092$ & $\mathrm{r}=0.061$ & $\mathrm{r}=0.090$ & $\mathrm{r}=0.063$ \\
\hline & $p=0.796$ & $p=0.134$ & $p=0.315$ & $p=0.140$ & $p=0.305$ \\
\hline \multirow[t]{2}{*}{ Superficial spreading infiltration } & $\mathrm{r}=0.037$ & $\mathrm{r}=0.035$ & $\mathrm{r}=0.154$ & $\mathrm{r}=0.060$ & $\mathrm{r}=0.064$ \\
\hline & $p=0.541$ & $p=0.570$ & $p=0.012$ & $p=0.325$ & $p=0.295$ \\
\hline \multirow[t]{2}{*}{ Resection margin } & $\mathrm{r}=0.035$ & $\mathrm{r}=0.091$ & $\mathrm{r}=0.139$ & $\mathrm{r}=0.099$ & $\mathrm{r}=0.044$ \\
\hline & $p=0.571$ & $p=0.136$ & $p=0.022$ & $p=0.104$ & $p=0.471$ \\
\hline \multirow[t]{2}{*}{ Perineural invasion } & $\mathrm{r}=0.156$ & $\mathrm{r}=0.026$ & $\mathrm{r}=0.055$ & $\mathrm{r}=0.009$ & $\mathrm{r}=0.101$ \\
\hline & $p=0.020$ & $p=0.695$ & $p=0.412$ & $p=0.896$ & $p=0.132$ \\
\hline \multirow[t]{2}{*}{ Lymphatic permeation } & $\mathrm{r}=0.065$ & $\mathrm{r}=0.090$ & $\mathrm{r}=0.025$ & $\mathrm{r}=0.170$ & $\mathrm{r}=0.013$ \\
\hline & $p=0.312$ & $p=0.163$ & $p=0.701$ & $p=0.008$ & $p=0.838$ \\
\hline \multirow[t]{2}{*}{ Microvascular invasion } & $\mathrm{r}=0.097$ & $\mathrm{r}=0.134$ & $\mathrm{r}=0.041$ & $\mathrm{r}=0.071$ & $\mathrm{r}=0.097$ \\
\hline & $p=0.154$ & $p=0.048$ & $p=0.548$ & $p=0.293$ & $p=0.151$ \\
\hline \multirow[t]{2}{*}{ Portal vein invasion } & $\mathrm{r}=0.004$ & $\mathrm{r}=0.002$ & $\mathrm{r}=0.179$ & $\mathrm{r}=0.019$ & $\mathrm{r}=0.017$ \\
\hline & $p=0.943$ & $p=0.972$ & $p=0.003$ & $p=0.757$ & $p=0.786$ \\
\hline \multirow[t]{2}{*}{ Lymph node metastases } & $\mathrm{r}=0.130$ & $\mathrm{r}=0.167$ & $\mathrm{r}=0.133$ & $\mathrm{r}=0.127$ & $\mathrm{r}=0.002$ \\
\hline & $p=0.033$ & $p=0.006$ & $p=0.029$ & $p=0.037$ & $p=0.980$ \\
\hline
\end{tabular}


a high rate of liver metastases, local recurrence, peritoneal dissemination, and lymph node metastases even if the tumor is completely resected (25). Furthermore, we found that there was a significant correlation between node-positive EBDCs and postoperative local recurrence, liver metastasis, peritoneal dissemination, and lymph node metastasis. Therefore, treatment failures, including locoregional failures and distant relapses, were common and adjuvant therapy may increase the rate of patient survival by reducing the effects of locoregional disease and improving systemic control (26).

Unfortunately, adjuvant chemotherapy has not yet been demonstrated to have a substantial survival benefit in postoperative cholangiocarcinoma patients. Available literature mainly consists of uncontrolled and often conflicting institutional series and registry analyses, although these studies do seem to favor an adjuvant approach $(27,28)$. In this study, our study cohort was composed solely of patients with curative resectable EBDCs. Although we did include EBDC patients from earlier records in the observation group, overall survival in the observation group was lower than in the adjuvant S-1 treatment group. Moreover, in subgroup analysis, adjuvant S-1 treatment showed a favorable HR in postsurgical patients who displayed symptoms for lymph node metastases and microvascular invasion. Therefore, it can be hypothesized that $\mathrm{S}-1$ adjuvant chemotherapy may be a viable option for treatment, depending on the profile of the patients.

However, this study was subject to several potential limitations. It is a retrospective cohort review of patients undergoing surgical resection that included only post-surgery patients in the study cohort. This design inherently represents a degree selection bias, due to the selection of patients chosen to undergo surgical management. This is particularly the case for, patients more whose records were older than 15 years and for whom we could not clearly determine how they receive adjuvant S-1 treatment. On the other hand, patients with postsurgical lymph node metastases were mainly administered S-1 adjuvant chemotherapy according to the guidelines outlined by our expert team. Undoubtedly, the current study was a very pertinent topic in our EBDC patient population, with the major question being which patients should be treated by adjuvant S-1 chemotherapy and how it should be conducted. Several clinical trials are now on-going and are either still recruiting patients or waiting to present their results. ACTICCA-1 is a randomized, multidisciplinary, and multinational phase III trial. It will evaluate the efficacy of gemcitabine and cisplatin, in terms of disease-free survival, in bile duct carcinoma patients after complete surgical resection. Two different patient cohorts will be included: the cholangiocarcinoma and the gallbladder carcinoma cohort. The French PRODIGE-12 study, which is evaluating the effects of gemcitabine and oxaliplatin and the British BILCAP study, examining capecitabine, are the two clinical trials which have not published their data up to this point. BILCAP is a multicenter, prospective, randomized phase III trial examining the role of adjuvant chemotherapy, using oral fluoropyrimidine (capecitabine), in biliary tract cancer patients after a potentially curative surgical resection. It has already completed accrual and the study results are expected to be published in the near future. In addition, there are two further ongoing studies in Japan. The BCAT (registration UMIN-CTR; ID UMIN000000820) study, which has already been closed, attempts to evaluate the effect of gemcitabine monotherapy when compared to surgical therapy. The other trial is the ASCOT (registration UMIN-CTR; ID UMIN000011688) study, which is still recruiting participants, and aims to evaluate the role of S-1 chemotherapy when compared primary surgical therapy. The final publication of these studies is eagerly anticipated, both due to their relevance for EBDC patients, and due the study design, setting, participants, and study methodology used in these studies, which could help improve subsequent research.

In conclusion, lymph node metastasis was clearly identified as a prognostic indicator of reduced overall survival in post-surgery EBDC patients. Locoregional recurrence was the most common type of failure and there was a significant correlation between node-positive EBDC and postoperative local recurrence, liver metastasis, and postoperative peritoneal dissemination. Therefore, we recommend adjuvant S-1 therapy in patients with lymph node metastases or microvascular invasion.

\section{Conflict of Interests}

The Authors declare no Conflict of Interests for this article.

\section{Acknowledgments}

This work was supported by the Kochi organization for medical reformation and renewal grants.

\section{References}

1 de Groen PC, Gores GJ, LaRusso NF, Gunderson LL and Nagorney DM: Biliary tract cancers. N Engl J Med 341: 13681378, 1999.

2 Edge SB: AJCC Cancer Staging Manual. 7th edn. New York: Springer, 2010

3 Khan SA, Thomas HC, Davidson BR and Taylor-Robinson SD: Cholangiocarcinoma. Lancet 366: 1303-1314, 2005.

4 Murakami Y, Uemura K, Sudo T, Hashimoto Y, Kondo N, Nakagawa N, Muto T, Sasaki H, Urabe K and Sueda T: Perineural invasion in extrahepatic cholangiocarcinoma: prognostic impact and treatment strategies. J Gastrointest Surg 17: 1429-1439, 2013.

5 Anderson C and Kim R: Adjuvant therapy for resected extrahepatic cholangiocarcinoma: a review of the literature and future directions. Cancer Treat Rev 35: 322-327, 2009. 
6 Takada T, Nimura Y, Katoh H, Nagakawa T, Nakayama T, Matsushiro T, Amano $\mathrm{H}$ and Wada $\mathrm{K}$ : Prospective randomized trial of 5-fluorouracil, doxorubicin, and mitomycin $\mathrm{C}$ for nonresectable pancreatic and biliary carcinoma: multicenter randomized trial. Hepatogastroenterology 45: 2020-2026, 1998.

7 Neoptolemos JP, Moore MJ, Cox TF, Valle JW, Palmer DH, McDonald AC, Carter R, Tebbutt NC, Dervenis C, Smith D, Glimelius B, Charnley RM, Lacaine F, Scarfe AG, Middleton MR, Anthoney A, Ghaneh P, Halloran CM, Lerch MM, Oláh A, Rawcliffe CL, Verbeke CS, Campbell F and Büchler MW; European Study Group for Pancreatic Cancer: Effect of adjuvant chemotherapy with fluorouracil plus folinic acid or gemcitabine $v s$. observation on survival in patients with resected periampullary adenocarcinoma: the ESPAC-3 periampullary cancer randomized trial. JAMA 308: 147-156, 2012.

8 Kaplan EL and Meier P: Nonparametric estimation from incomplete observations. J Am Stat Assoc 53: 457, 1958.

9 Cox DR: Regression models and life tables. J R Stat Soc 187: 34B, 1972.

10 Gwak HK, Kim WC, Kim HJ and Park JH: Extrahepatic bile duct cancers: surgery alone versus surgery plus postoperative radiation therapy. Int J Radiat Oncol Biol Phys 78: 194-198, 2010.

11 Ebata T, Yokoyama Y, Igami T, Sugawara G, Mizuno T and Nagino M: Review of hepatopancreatoduodenectomy for biliary cancer: an extended radical approach of Japanese origin. J Hepatobiliary Pancreat Sci 21: 550-555, 2014.

12 Mansour JC, Aloia TA, Crane CH, Heimbach JK, Nagino M and Vauthey JN: Hilar cholangiocarcinoma: expert consensus statement. HPB (Oxford) 17: 691-699, 2015.

13 Yoshitomi H, Miyakawa S, Nagino M, Takada T and Miyazaki M: Updated clinical practice guidelines for the management of biliary tract cancers: revision concepts and major revised points. J Hepatobiliary Pancreat Sci 22: 274-278, 2015.

14 American Cancer Society. [Accessed October 18, 2017] Cancer Facts \& Figures. 2011. http://www.cancer.org/Research/ CancerFactsFigures/CancerFactsFigures/cancer-facts-figures2011

15 Siegel R, Ma J, Zou Z and Jemal A: Cancer statistics, 2014. CA Cancer J Clin 64: 9-29, 2014.

16 Nagahashi M, Shirai Y, Wakai T, Sakata J, Ajioka Y, Nomura T, Tsuchiya $\mathrm{Y}$ and Hatakeyama K: Depth of invasion determines the postresectional prognosis for patients with $\mathrm{T} 1$ extrahepatic cholangiocarcinoma. Cancer 116: 400-405, 2010.

17 Blom D and Schwartz SI: Surgical treatment and outcomes in carcinoma of the extrahepatic bile ducts: the University of Rochester experience. Arch Surg 136: 209-215, 2001.

18 Kamposioras K, Anthoney A, Fernández Moro C, Cairns A, Smith AM, Liaskos C and Verbeke CS: Impact of intrapancreatic or extrapancreatic bile duct involvement on survival following pancreatoduodenectomy for common bile duct cancer. Br J Surg 101: 89-99, 2014.
19 Jang JY, Kim SW, Park DJ, Ahn YJ, Yoon YS, Choi MG, Suh $\mathrm{KS}$, Lee KU and Park YH: Actual long-term outcome of extrahepatic bile duct cancer after surgical resection. Ann Surg 241: 77-84, 2005.

20 Sakamoto Y, Kosuge T, Shimada K, Sano T, Ojima H, Yamamoto J, Yamasaki S, Takayama T and Makuuchi M: Prognostic factors of surgical resection in middle and distal bile duct cancer: an analysis of 55 patients concerning the significance of ductal and radial margins. Surgery 137: 396-402, 2005.

21 Nakeeb A, Pitt HA, Sohn TA, Coleman J, Abrams RA, Piantadosi S, Hruban RH, Lillemoe KD, Yeo CJ and Cameron JL: Cholangiocarcinoma. A spectrum of intrahepatic, perihilar, and distal tumors. Ann Surg 224: 463-473, 1996.

22 Fong Y, Blumgart LH, Lin E, Fortner JG and Brennan MF: Outcome of treatment for distal bile duct cancer. Br J Surg 83: 1712-1715, 1996.

23 Choi SB, Park SW, Kim KS, Choi JS and Lee WJ: The survival outcome and prognostic factors for middle and distal bile duct cancer following surgical resection. J Surg Oncol 99: 335-342, 2009.

24 Murakami Y, Uemura K, Hayashidani Y, Sudo T, Hashimoto Y, Ohge H, and Sueda T: Prognostic significance of lymph node metastasis and surgical margin status for distal cholangiocarcinoma. J Surg Oncol 95: 207-212, 2007.

25 Fawcett TJ, Parry ML and Blanck G: A novel approach to evaluating cancer driver gene mutation densities: cytoskeletonrelated gene candidates. Cancer Genomics Proteomics 12: 283290, 2015.

26 Purdy M, Kokki M, Anttila M, Aspinen S, Juvonen P, Selander T, Kokki H, Pulkki K and Eskelinen M: Does post-surgery placement of rectus sheath block analgesia alter the oxidative stress biomarker 8-OHdG concentrations: a randomised trial of patients with cancer and benign disease. Cancer Genomics Proteomics 13: 239-244, 2016.

27 Nakeeb A and Pitt HA: Radiation therapy, chemotherapy and chemoradiation in hilar cholangiocarcinoma. HPB (Oxford) 7(4): 278-282, 2005.

28 Bolm L, Kaesmann L, Bartscht T, Schild SE and Rades D: Outcomes after radio(chemo)therapy for non-metastatic bile duct cancer. In Vivo 31: 117-120, 2017. 